\title{
Review of Trichobezoar and its Clinical Relevance with A Special Reference to an Unusual Presentation of Rapunzel Syndrome, A Double Impacted Trichobezoar
}

\author{
Kapil Rampal *1, Devendra Prajapati ${ }^{2}$, Meghna Sharma ${ }^{3}$. \\ ${ }^{* 1}$ Senior Resident surgery Deen Dayal Upadhyaya Hospital ,Hari Nagar, New Delhi, India. \\ ${ }^{2}$ Senior Resident surgery Deen Dayal Upadhyaya Hospital ,Hari Nagar, New Delhi, India. \\ ${ }^{3}$ M edical officer PCM S-1, CHC Kasel , Tarn Taran Punjab, India.
}

\section{ABSTRACT}

Bezoars are collections of interwoven organic or inorganic matter found in the alimentary canal. Rapunzel syndrome represents a rare presentation of trichobezoar where the tail of main gastric bezoar extends into the lumen of small intestine. We present the case of a 09 year old female child who presented to us as a case of acute intestinal obstruction as a rare manifestation of Rapunzel syndrome, with migration of gastric bezoar into the small intestine. Successful surgical retrieval was performed in emergency scenario and the patient had an uneventful recovery.

KEY WORDS: Trichobezoar, Rapunzel syndrome, Trichotillomania, Trichophagia, Intestinal obstruction, abdominal mass.

Address for correspondence: Dr. Kapil Rampal, Senior Resident, Deen Dayal Upadhyaya Hospital, Hari Nagar, New Delhi- 110064, India. Telephone: +919718379366/ +919650109599

E-Mail: balkarankapil@gmail.com

\begin{tabular}{|c|c|}
\hline \multicolumn{2}{|r|}{ Online Access and Article Informtaion } \\
\hline \multirow{3}{*}{ Quick Response code } & International Journal of Integrative Medical Sciences \\
\hline & www.imedsciences.com \\
\hline & 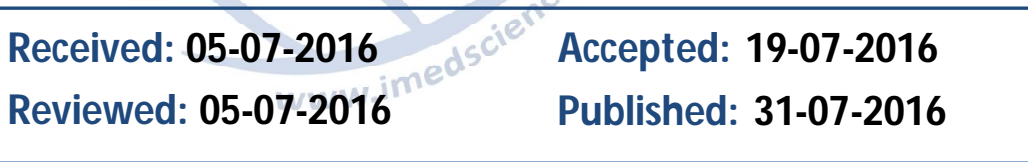 \\
\hline Source of Funding: Self & Conflicts of interest: None \\
\hline
\end{tabular}

\section{BACKGROUND}

The term bezoar is derived from Persian word 'bedzehr' or 'padzehr' meaning anti-poison. Bezoars medically are a collection of tightly interwoven indigestible matters present in the alimentary canal. Subsequent nomenclature is governed by the constituent matter of the bezoar as hairs- trichobezoar, vegetables- phytobezoar, milk and curd- lactobezoar. Rare forms of bezoars as stone and fur bezoars have also been reported in literature. Trichobezoars are the most common of the bezoars comprising more than $50 \%$ of the bezoars described in the literature. Rapunzel syndrome has been described in the literature as a rare presentation of trichobezoar where the tail of main gastric bezoar extends through the pylorus to small intestine causing partial or complete gastric obstruction [1]. It results from a psychiatric illness trichotillomania in which the patient achieves pleasure and fulfillment through pulling and ingesting hair(trichophagia). The problem is almost exclusively seen in young females who may also show accompanying features of depression, anxiety or poor self image and esteem [2,3].

Etiopathogenesis: Bezoars are retained collections of indigestible foreign material that accumulate in the GI tract. Stomach is the most common location, but they can occur in the esophagus, small intestine, and rectum. Altered 
gastric physiology with impaired gastric emptying and decreased acid production has been incriminated in bezoar formation. These changes are usually consequences of previous gastric surgery, which is present in $70 \%$ to $94 \%$ of patients with bezoar formation. Vagotomy with pyloroplasty or antrectomy is a predisposing factor for bezoar formation. Patients with gastroparesis, diabetes, end-stage renal disease, and prolonged mechanical ventilation are at increased risk for bezoars. In some patients, ingestion of large amounts of indigestible material is the only risk factor for bezoar formation.There are four types of bezoars: phytobezoars, trichobezoars, pharmacobezoars, and lactobezoars. Phytobezoars are the most frequent type of bezoar. These foods are composed of large amounts of nondigestible fiber such as cellulose, lignin, and fruit tannin. A high concentration of tannins, exposed to gastric acid, can form a coagulum leading to bezoar formation. Phytobezoars are typically dark brown, black, or green when visualized by endoscopy. Trichobezoars are composed of hair or hair-like fibers. They are observed most commonly in children and women younger than 30 years. Frequently, these patients have underlying mental retardation or psychiatric disorders.

Trichotillomania is an impulse control disorder characterized by the repeated urge to pull out scalp and body hair. Trichophagia is the compulsion to eat or chew on hair. These behavioral disorders are associated with trichobezoar formation. Hair and fibers in the stomach become trapped in gastric folds. Trichobezoars are typically black regardless of the color of the hair ingested because of enzymatic oxidation of gastric acid on the hair fibers. Some gastric trichobezoars have a long extension of hair that trails into the duodenum, a condition known as the Rapunzel syndrome. It has been reported to cause jaundice and pancreatitis as a result of obstruction of the ampulla of Vater by hair.

Pharmacobezoars are composed of medications or vitamins. Resin-coated, extended-release products or other products designed to resist digestion are most often the nidus for this type of bezoar formation. Pharmacobezoars can result in reduced medication efficacy because the active agent is trapped in the bezoar and cannot be absorbed. Alternatively, toxicity can result when the previously bound active agent is released in excessive amounts. Fatality secondary to pharmacobezoars has been reported. Lactobezoars are a compact mass of undigested milk concretions located within the Gl tract of infants and toddlers. These rare bezoars have been linked to nearly every commercially available infant formula and breast and cow milk [4].

Signs and symptoms: The most common symptom present in $80 \%$ of patients with bezoars is vague epigastric discomfort. Other symptoms include nausea, vomiting, anorexia, early satiety, and weight loss. If a bezoar reaches a large size and is present for a prolonged period, it may cause pressure necrosis and ulcers. Ulceration can lead to bleeding or obstruction. Once in the small bowel, bezoars most commonly result in obstruction. Physical examination is often unrevealing. Occasionally, a palpable mass may be present. Patients with trichotillomania and trichobezoars may have patchy baldness [4].

Diagnosis: Bezoars can be demonstrated on plain films and computed tomography scans. Barium studies reveal a gastric filling defect. Radiography may reveal only $25 \%$ of bezoars. Endoscopy is the gold standard for the diagnosis of bezoars. The endoscopic appearance of phytobezoars consists of an amorphous mass of brown, green, or black material. Trichobezoars are black, hard, and concrete-like. Pharmacobez-oars contain whole pills or pill fragments. Lactobezoars are most commonly identified on contrast-enhanced upper $\mathrm{GI}$ series or ultrasound [5].

Case presentation: A 09 year old female child who presented to us in the surgical emergency with pain abdomen, recurrent episo des of bilious vomiting, obstipation and distension of abdomen of 03 days duration. There was no history of fever, loose motions, gastrointestinal bleed, similar episodes in past or the presence of any medical co-morbidities. On examination the child had tachycardia with a pulse rate of 110/ minute. The child was afebrile, had a normal blood pressure and a normal hydration status. 
Fig. 1: Showing the Supine and upright $X$ ray abdomen.

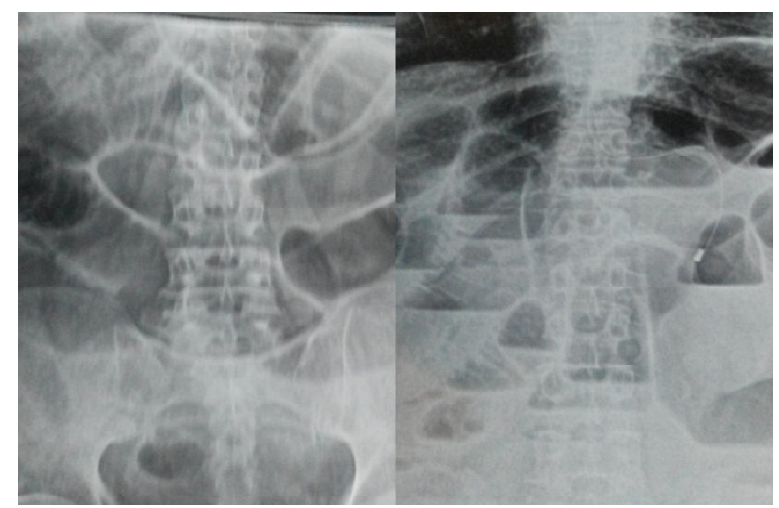

Fig. 2: Showing the Large sized trichobezoar.

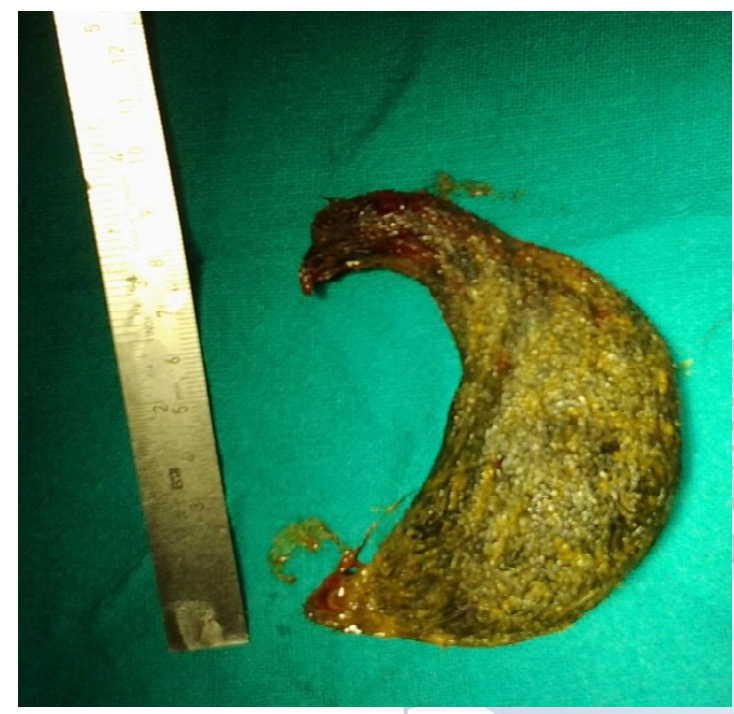

Fig. 3: Showing smaller trichobezoar.

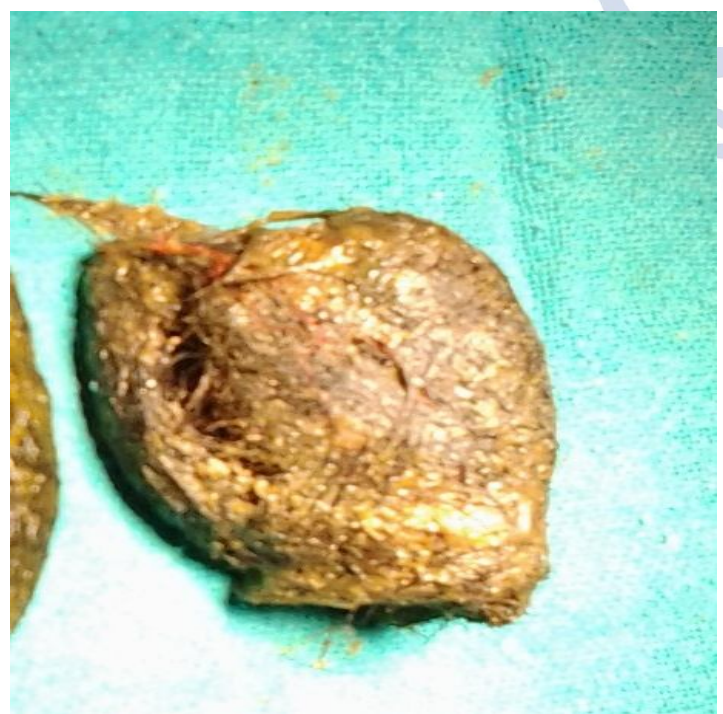

Per abdomen examination showed marked distension, no features of peritonitis, the bowel sounds were absent. Digital rectal examination showed an empty rectum. Supine and upright $X$ ray abdomen (Figure 1 ) showed dilated small bowel loops. Ultrasound abdomen suggested grossly dilated small bowel loops with poor peristalsis. The child was resuscitated with intravenous fluids and taken up for emergency exploratory laparotomy as a case of acute intestinal obstruction.

Per operative findings included minimal ascites, large sized trichobezoar measuring $10 \mathrm{~cm} \mathrm{X} 4.5$ $\mathrm{cm}$ with a distal tailing of hair strands (Figure 2) stuck in jejunum $20 \mathrm{~cm}$ distal to the duodenojejunal flexure with collapsed proximal jejunal loop, smaller trichobezoar measuring $4.5 \mathrm{~cm} \times 3.5 \mathrm{~cm}$ with proximal tailing of hair strands (Figure3) stuck in terminal ileum $10 \mathrm{~cm}$ proximal to the ileocaecal junction with collapsed distal ileum and the large bowel. Proximal larger trichobezoar had assumed the shape of stomach confirming its gastric origin.

Both bezoars were retrieved through separate enterotomies considering the risks involved with in situ manipulation of unknown foreign bodies in trying to achieve a single enterotomy. Both enterotomies were repaired primarily. Post operative period was uneventful. In the post operative period the child confessed to ingestion of hair stuck in the comb of herself and her mother over a period of 02 years.

\section{DISCUSSION}

The common presentation of tichobezoar is in a young female with an underlying psychiatric disorder. Trichobezoar in itself is a rare entity as its prevalence in patients with trichophagia has been described to be less than $1 \%$ [6]. Stomach is the most common site for trichobezoar formation as the ingested hair strands escape peristaltic propulsion and are retained in prepyloric gastric folds and persistent peristalsis enmeshes them into an interwoven ball. Studies have linked trichobezoar to childhood neglect, abuse, mental retardation or bereavement $[7,8]$. $M$ ajority of the cases are detected late due to an ignorant patient and rarity of the condition resulting in a low index of suspicion amongst the physicians.

The patient usually presents with a palpable abdominal mass (87.7\%), abdominal pain (70.2\%), nausea and vomiting (64.9\%), weakness and weight loss (38.1\%), obstruction or diarrhea (32\%) and low hemoglobin (62\%) [9].

Treatment modalities available include endoscopic therapy and surgical intervention. 
Endoscopic therapy is more effective for soft bezoars like phytobezoars (vegetable) and lactobezoars (milk and curd). Use of specialized bezotomes and bezotriptors that pulverize and fragment the bezoars has been described in literature [10]. However technical intricacies involved and limited availability of these tools offset their advantages over a formal laparotomy. Enzymatic dissolution of bezoars has also been described with cellulose, papain (meat tenderizer), $\mathrm{N}$-acetylcysteine, and CocaCola(carbonated beverage) [11-13].

\section{CONCLUSION}

Trichobezoar is a rare but still under diagnosed clinical condition. This should be considered as differential diagnosis in a young female patient presenting with abdominal mass, intestinal obstruction or pain abdomen and a specific history of pica should be obtained. Patient have associated psycho-social issues that need to be addressed to avoid a recurrence. Also as cases of trichobezoar and further Rapunzel syndrome themselves being rare, our case presentation of a gastric trichobezoar migrating and causing small intestinal obstruction due to double impaction with its satellite tuft of hair becomes still rarer.

\section{Conflicts of interest: None}

\section{REFERENCES}

[1]. Phillips MR, Zaheer S, Drugas GT: Gastric trichobezoar: case report and literature review. Mayo Clin Proc 1998;73:653-656.

[2]. Diefenbach GJ, Reitman D, Williamson DA: Trichotillomania: a challenge to research and practice. Clin Psychol Rev 2000;20:289-309.

[3]. Carr JR, Sholevar EH, Baron DA: Trichotillomania and trichobezoar: a clinical practice insight with report of illustrative case. J Am Osteopath Assoc 2006;106:647-652.

[4]. Shackelford's Surgery of the Alimentary Tract, $6^{\text {th }}$ Edition.

[5]. DuBose 5th TM, Southgate WM , Hill G]: Lactobezoars: A patient series and literature review. Clin Pediatr (Phila) 2001;40:603-606.
[6]. Irving PM, Kadirkamanathan SS, Priston AV, Blanshard C. Education and imaging. Gastrointestinal: Rapunzel syndrome. J Gastroenterol Hepatol 2007;22:2361.

[7]. Gockel I, Gaedertz C, Hain HJ, Winckelmann U, Albani M I, Lorenz D. [The Rapunzel syndrome: rare manifestation of a trichobezoar of the upper gastrointestinal tract.] Chinurg 2003;74:753-756.

[8]. Sharma NL, Sharma RC, Mahajan VK, Sharma RC, Chauhan D, Sharma AK. Trichotillomania and trichophagia leading to trichobezoar. J Dermatol 2000;27:24-26.

[9]. Gupta Naik S, Naik S, Chaudhary AK, Jain P, Sharma A. Rapunzel Syndrome Reviewed and Redefined. Dig Surg 2007;24:157-161.

[10]. Wang YG, Seitz U, Li ZL, Soehendra N, Qiao XA. Endoscopic management of huge bezoars. Endoscopy 1998;30:371-374.

[11].Walker-Renard P. Update on the medical management of phytobezoars. Am J Gastroenterol 1993;88:1663-1666.

[12]. Zarling EJ, M oeller DD. Bezoar therapy: Complications using Adolph's meat tenderizer and alternatives from literature review. Arch Intern Med 1981;141:1669-1670.

[13]. Sechopoulos P, Robotis JF, Rokkas T. Gastric bezoar treated endoscopically with a carbonated beverage: Case report. Gastrointest Endosc 2004;60:662664.

\footnotetext{
How to cite this article:

Kapil Rampal, Devendra Prajapati, M eghna Sharma. Review of Trichobezoar and its Clinical Relevance with A Special Reference to an Unusual Presentation of Rapunzel Syndrome, A Double Impacted Trichobezoar Int J Intg M ed Sci 2016;3(7):361-364. DOl: 10.16965/ijims.2016.139
} 